\title{
A Prediction Model using Machine-Learning Algorithm for Assessing Optimal Intrathecal Hyperbaric Bupivacaine Dose during Cesarean Section
}

\section{Chang-na Wei}

Department of Anesthesia,Jiaxing Affiliated Women and Children Hospital

\section{Li-ying Wang}

department of Anesthesia,Jiaxing University Affiliated Women and Children Hospital

\section{Xiang-he Zhou}

department of Anesthesia,Affiliated hospital of Jiaxing University

Qing-he Zhou ( $\sim$ jxxmxy@163.com )

Affiliated Hospital of Jiaxing University, Zhejiang Province

\section{Research article}

Keywords: Machine learning algorithm, Bupivacaine dosage, Cesarean section, Spinal anesthesia, Physical variables

Posted Date: October 6th, 2020

DOI: https://doi.org/10.21203/rs.3.rs-80984/v1

License: (c) (1) This work is licensed under a Creative Commons Attribution 4.0 International License. Read Full License

Version of Record: A version of this preprint was published at BMC Anesthesiology on April 14th, 2021. See the published version at https://doi.org/10.1186/s12871-021-01331-8. 
A Prediction Model using Machine-Learning Algorithm for Assessing Optimal Intrathecal Hyperbaric Bupivacaine Dose during Cesarean Section

Chang-na Wei, MD†, Li-ying Wang, MD $†$ Xiang-yang Chang, MD† and Qing-he Zhou, Ph.Dł

tDepartment of Anesthesia, Jiaxing University Affiliated Women and Children Hospital, Jiaxing, Zhejiang Province, China

¥Department of Anesthesia, Affiliated Hospital of Jiaxing University, Jiaxing, Zhejiang Province, China

Address correspondence to:

Qing-he Zhou, Ph.D., Director of Anesthesia Department, Affiliated Hospital of Jiaxing University, Zhejiang Province, China

Email: jxxmxy@163.com

Tel: +8613732573379

Fax $+860573-82059702$

Short Title: Machine learning algorithm for bupivacaine at cesarean 


\section{Abstract}

Background: The optimal intrathecal hyperbaric bupivacaine dosage for cesarean section is difficult to predetermine. The aim of this study was to develop a decision-support model using a machine-learning algorithm for assessing intrathecal hyperbaric bupivacaine dose based on physical variables during cesarean section.

Methods: Term parturients presenting for elective cesarean section under spinal anaesthesia were enrolled. Spinal anesthesia was performed at the $L 3 / 4$ interspace with $0.5 \%$ hyperbaric bupivacaine at dosages determined by the anesthesiologist. A spinal spread level between T4-T6 was considered the appropriate block level. We used a machine-learning algorithm to identify relevant parameters. The dataset was split into derivation (80\%) and validation (20\%) cohorts. An optimal decision-support model was developed for obtaining the regression equation between optimized intrathecal $0.5 \%$ hyperbaric bupivacaine volume and physical variables.

Results: A total of 684 parturients were included, of whom $516(75.44 \%)$ and 168 (24.56\%) had block levels between T4 and T6, and less than T6 or higher than T4, respectively. The appropriate block level rate was $75.44 \%$. In lasso regression, based on the principle of predicting a reasonable dose of intrathecal bupivacaine with fewer physical variables, the optimal model is " $\mathrm{Y}=0.5922+0.055117 * \mathrm{X}_{1}-0.017599 * \mathrm{X}_{2}$ " ( $\mathrm{Y}$ : bupivacaine volume; $\mathrm{X}_{1}$ : vertebral column length; $\mathrm{X}_{2}$ : abdominal girth), with $\lambda$ 0.055, MSE(mean square error) 0.0087 , and $R^{2} 0.807$.

Conclusion: After applying a machine-learning algorithm, we developed a decision model with $\mathrm{R}^{2} 0.8070$ and MSE due to error 0.0087 using abdominal girth and vertebral column length for predicting the optimized intrathecal $0.5 \%$ hyperbaric bupivacaine dosage during term cesarean sections.

Keywords: Machine learning algorithm; Bupivacaine dosage; Cesarean section; Spinal anesthesia; Physical variables 


\section{Background}

Cesarean delivery is routinely performed under spinal anesthesia with hyperbaric bupivacaine [1-3]. Bupivacaine provides an appropriate duration of anesthesia to perform cesarean delivery, and hyperbaric bupivacaine may ensure a more predictable block[4]. However, it is still a challenge for the anesthetist to achieve the optimal spinal spread for cesarean delivery[5]. Parturient variables, including age, height, weight, and body mass index (BMI) have been used to predict spinal spread, but results have been inconsistent $[6,7]$ Our previous study demonstrated that abdominal girth and vertebral column length correlated favorably with spread of intrathecal hyperbaric bupivacaine in term parturients. ${ }^{8}$ We further established an elemental regression equation between parturient vertebral column length, abdominal girth, and $0.5 \%$ hyperbaric intrathecal bupivacaine volume for $\mathrm{T} 5$ block level[9]. ${ }^{9}$ However, the sample size in these studies was relatively small and the accuracy of the regression equation needed further verification $[8,9]$

In recent years, there has been an advance in machine-learning algorithms in a number of fields including anesthesiology, which allowed large amounts of data for development of robust predictive analytics[10-13]. These were used to predict, inter alia, postinduction hypotension[13]. intraoperative hypotension[11], and length of hospital stay[14]. In our hospital, more than 1000 parturient women undergo cesarean section under spinal anesthesia annually. Based on the large number of sample sources, the purpose of this prospective study was to develop a decision-support model using a machine-learning algorithm for assessing optimized intrathecal hyperbaric bupivacaine dosage based on their physical variables at cesarean section.

\section{Methods}

\section{Patients}

The study protocol was approved by the Ethical Committee of Jiaxing Maternity and Child Health Care Hospital in May 2015 (2015-5), and pre-registered at 
http://www.chictr.org.cn/index.aspx (ChiCTR-OOC-16009149) in September 3, 2016. The informed consent was obtained from all participants. In this prospective observational study, 684 term parturient women who presented for elective cesarean section under spinal anesthesia were enrolled from October 2016 to November 2019. Inclusion criteria were American Society of Anesthesiologists (ASA) physical status II or III, age between 21 and 40 years, and gestational age over 37 completed weeks. Exclusion criteria were patient refusal, significant medical or obstetric comorbidities, multiple pregnancy, failed spinal anesthesia, contraindication to spinal anesthesia, discrepancy in spread between both midclavicular lines, or history of allergy to bupivacaine.

\section{Spinal anaesthesia}

The parturient fasted for $8 \mathrm{~h}$ and received $8 \mathrm{~mL} / \mathrm{kg}$ Ringer's lactate solution via peripheral intravenous access before spinal anesthesia. After entry to the operation room, the standard ASA monitoring was performed. Before spinal puncture, the $L 3 / 4$ interspace was confirmed by ultrasonic imaging. Spinal anesthesia was performed at the L3/4 interspace in the left lateral decubitus position with a midline approach; $0.5 \%$ hyperbaric bupivacaine was injected into the subarachnoid space over $10 \mathrm{~s}$ after free flow of cerebrospinal fluid had been obtained. The volume of $0.5 \%$ hyperbaric bupivacaine administered was based on the experience of the anesthesiologist. After these procedures, the woman were then rapidly placed in a supine position, with a right pelvic wedge placed to facilitate left uterine displacement. All anesthesia procedures were performed by the same attending physician, and the assessment of cephalad spread of spinal anesthesia was completed by another anesthetist who was blind to the measurement information about the woman.

Surgery commenced $15 \mathrm{~min}$ after intrathecal injection. If the parturient complained of severe pain (general Visual Analogue Scale $[\mathrm{VAS}] \geq 4$ ) during surgery, remifentanil was administered with micropump injection for rescue analgesia. If, based on the assessment of the anesthetist, the woman could not endure the surgery under spinal anesthesia, general anesthesia was performed. Hypotension was defined as systolic pressure values $<90 \mathrm{mmHg}$ 
or systolic pressure decreases $>30 \%$ and was treated with $6 \mathrm{mg}$ of ephedrine or $100 \mathrm{mg}$ norepinephrine intravenously. Bradycardia was defined as heart rate values $<55$ beats $/ \mathrm{min}$ and was treated with atropine $0.5 \mathrm{mg}$ intravenously.

\section{Outcome measures}

Abdominal girth was measured at the level of the umbilicus at the end of expiration when the parturient were placed supinely on the horizontal operating table. Vertebral column length was measured from the $\mathrm{C7}$ vertebra to the sacral hiatus (C7-SH). Cephalad spread of spinal anesthesia was assessed by testing for loss of pinprick discrimination in both midclavicular lines at $3 \mathrm{~min}$ intervals after intrathecal injection. The spread level at $15 \mathrm{~min}$ after intrathecal bupivacaine injection was used for the analysis. Spinal spread level between T4-T6 was considered the appropriate block level. The volume of intrathecal bupivacaine was recorded. Parturient demographic variables, including age, height, fundal height, and weight were recorded. Demographic variables, such as fetal biparietal diameter, were also recorded. As predictors for the machine-learning models, we included parturient age, height, fundal height, weight, abdominal girth, vertebral column length, and fetal biparietal diameter. The primary outcome was to obtain the regression equation between optimized intrathecal $0.5 \%$ hyperbaric bupivacaine dosage and physical variables.

\section{Decision-support model analysis}

Machine-learning algorithm was performed by Python 3.7.1. The objective function of lasso regression is:

$$
\min _{\omega}\|y-X \omega\|_{2}^{2}+\lambda\|\omega\|_{1}
$$

In the function, $\lambda$ is a parameter that controls the complexity of the model. With lasso regression, controlling the parameter of $\lambda$ can result in a sparse solution: the coefficient of unimportant features will be assigned to 0 , and the important features will be highlighted according to the weight value so as to achieve the importance ranking of features, and further control the complexity of the algorithm according to the selected input features.

Therefore, based on the lasso regression algorithm, our study explored the relationship 
between the seven individual physical variables listed above and bupivacaine dosage, then obtained the regression equation between optimized intrathecal $0.5 \%$ hyperbaric bupivacaine dosage and the physical variables.

The statistical model strives to predict an optimal intrathecal bupivacaine dosage with fewer physical variables. $R^{2}$ is the determination coefficient, which is the degree of fitting for the obtained regression equation. Mean-square error (MSE) is a single value that provides information about the goodness fit of the regression line. The smaller the MSE value, the better the fit, as smaller values imply smaller magnitudes of error. The data set (term parturient with appropriate block level) was split into derivation (80\%) and validation (20\%) cohorts. The derivation cohort parturients were used to derive for the prediction model, and the validation cohorts were used to validate the model. We gradually increased the number of independent variables from 2 to 5 (physical variables), optimized the $\lambda$ value, and balanced the value of $R^{2}, M E S$, and the number of independent variables.

All statistical analyses were performed with SPSS version 19 (IBM Corp). We presented the data as means \pm standard deviations (SDs) or numbers (\%) as appropriate. We tested quantitative data by using the independent $t$-test. A two-tailed $p$ value $<0.05$ denoted statistical significance.

\section{Results}

A total of 684 parturients were included in this study, of whom 516 had a block level between T4 and T6 (appropriate block level) and 168 had a block level lower than T6 or higher than T4 (inappropriate block level). The appropriate block level rate was $75.44 \%$. The spread level of 69 parturients was less than T6 and 99 parturients higher than T4. Compared with those with appropriate block levels, weight and abdominal girth were greater in the group with inappropriate block levels ( $p<0.05$ and $p<0.01$, respectively); there was no obvious difference in bupivacaine dosage and other physical variables in those women with appropriate and inappropriate block levels (Table 1).

A total of 412 term parturients were used to derive for the prediction model. In lasso 
regression, when the physical variables included in the equation increased from 2 to $5, R^{2}$ increased from 0.8070 to 0.81325 , and MSE decreased from 0.0087 to 0.00844 , both with little changed.

On decision-tree analysis, vertebral column length and abdominal girth were the top two physical performance variables with respect to the optimal dosage of intrathecal bupivacaine; the optimal model is " $\mathrm{Y}=0.5922+0.055117 * \mathrm{X}_{1}-0.017599 * \mathrm{X}_{2}$ " $(\mathrm{Y}$ : $0.5 \%$ bupivacaine volume ; $X_{1}$ : vertebral column length; $X_{2}$ :abdominal girth), with the $\lambda 0.055$, MSE 0.0087 and $R^{2} 0.807$ (Table 2). The remaining 104 participants were used to validate the model. The actual bupivacaine volume was $1.94 \pm 0.21 \mathrm{~mL}$, with a predicted bupivacaine volume of $1.95 \pm 0.19$ $\mathrm{mL}(p>0.05) ; \mathrm{R}^{2}$ of all women during the validation was above 0.8 , indicating that the model was reliable (Figs 1 and 2).

In the current study, 282 parturients (41.23\%) were found to develop hypotension during cesarean section and were treated with ephedrine and/or norepinephrine.

\section{Discussion}

In this prospective, observational study, after applying a machine-learning algorithm, we developed the decision model: $Y=0.5922+0.055117 * X_{1}-0.017599 * X_{2}(Y: 0.5 \%$ hyperbaric bupivacaine volume; $X_{1}$ : vertebral column length; $X_{2}$ : abdominal girth), with the $\lambda 0.055$, MSE 0.0087 , and $R^{2} 0.807$. On decision-tree analysis, vertebral column length and abdominal girth were the top two performance physical variables with respect to intrathecal bupivacaine dosage.

According to the data type of this study, multiple linear regression, ridge regression, and lasso regression are usually used for data analysis. Multiple linear regression has the characteristics of simple construction, easy implementation, and low operation complexity, but the model is prone to over-fitting. To control the complexity of the model, penalties or other constraints are often added to the model; that is, regularization techniques. Ridge regression and lasso regression are currently popular regularization regression techniques. The goal of the two is the same; that is, to minimize the sum of squared residuals, but the 
constraints on the regression coefficients are different. Ridge regression can effectively solve the problem of over-fitting but, as the value changes, its feature coefficients become uniformly small, making it impossible to discern the importance of each feature. Lasso regression, however, effectively solves the over-fitting problem; it can obtain a sparse solution by controlling the parameters: the coefficients of unimportant features will be assigned to 0 , and the important features will be highlighted according to the weight value, so as to achieve the importance ranking of features, and further control the complexity of the algorithm according to the selected input features.

In the present study, according to the results of parameter selection of lasso regression and model evaluation for different parameters, when the physical variables included in the equation increased from 2 to $5, M S E$ and $R^{2}$ were not obviously increased. On decision-tree analysis, vertebral column length and abdominal girth were the top two performance physical variables with respect to optimal intrathecal bupivacaine dosage. Over one hundred term parturients were used to validate the model, and $R^{2}$ of all patients during the validation was above 0.8 , indicating that the model was reliable.

Previous studies reported that the median satisfactory block height for the loss of pinprick discrimination during spinal anesthesia for cesarean section was T5, and the interquartile range (IQR) was from T4-T6[15]. In our previous study, we set T5 as the appropriate spinal spread level and found the vertebral column length and abdominal girth to be the top two performance physical variables[9]. In the current study, we set appropriate spinal spread levels for the loss of pinprick discrimination as T6, T5, and T4 at 15 min after intrathecal injection, and also found vertebral column length and abdominal girth to be the top two performance physical variables. We need to note that the regression model obtained in this study is more clinically valuable.

Studies have proven that, in pregnant women, soft tissues may migrate inward into the vertebral canal[16], and engorgement of the extradural venous plexus occurs when pregnant women are in the supine position because of obstruction of the inferior vena cava by the 
enlarged uterus[17,18].Thus, an increased abdominal girth in the parturient causes a decrease[20].In lumbosacral cerebrospinal fluid volume. Carpenter et al[19]. reported that lumbosacral cerebrospinal fluid volume is the main determinant of the spread of spinal anesthesia. Our recent study showed that abdominal girth and dorsosacral distance were correlated with lumbosacral cerebrospinal fluid volume Therefore, the maternal abdominal girth and vertebral length may have a predictive effect on the spinal spread due to their effect on the volume of the lumbosacral cerebrospinal fluid.

Previous studies reported incidences of hypotension varying from $1.9 \%$ to $71 \%[21,22]$. In the present study, hypotension during cesarean section occurred in $41.23 \%$ of participants and was treated with ephedrine and/or norepinephrine. Most of these patients were term parturients with appropriate spinal spread level. Thus, when performing cesarean section under spinal anesthesia, we must strictly monitor the patient's hemodynamic status, irrespective of whether or not the anesthesia block level is in the appropriate range.

This study has several limitations. First, only the spread level at $15 \mathrm{~min}$ after intrathecal bupivacaine injection was used for the analysis. We know that the spinal spread changes over time. Second, further increasing the sample size may result in a more accurate regression equation. Despite these limitations, the current machine-learning algorithm provides new insights into the potential impact of controversial parameters.

\section{Conclusion}

In conclusion, in the current study, after applying a machine-learning algorithm, we developed a decision model with the coefficient of determination 0.807 and the mean of squares due to error 0.0087 , using two physical variables for predicting the optimal intrathecal $0.5 \%$ hyperbaric bupivacaine dosage during cesarean section in term parturients. Among the parturient physical variables, abdominal girth and vertebral column length were the two most significant factors, which can be used for predicting the intrathecal bupivacaine dosage during cesarean section. 


\section{Abbreviation}

MSE: Mean-square Error; $\mathrm{R}^{2}$ : Coefficient of determination; BMI: body mass index; ASA: American Society of Anesthesiologists;VAS: Visual Analogue Scale; SPSS: Statistic Package for Social Science; IQR: inter quartile range

\section{Declarations}

\section{Consent for publication}

Not applicable.

\section{Availability of data and materials}

The datasets used and/or analyzed during the current study are available from the corresponding author on reasonable request.

\section{Competing interests}

The authors declare that they have no competing interests.

\section{Funding}

The study was funded by Technology Bureau of Jiaxing, Zhejiang Provience(2015C23023) and Jiaxing Key Subject of Anesthesiology.

\section{Acknowledgements}

We would like to thank Ying-chun Ren for the analysis of data and and Wei Deng for discussion of the planning in current study. The study protocol was pre-registered at http://www.chictr.org.cn/index.aspx (ChiCTR-OOC-16009149) in September 3, 2016.

\section{References}

1. Norris MC. Patient variables and the subarachnoid spread of hyperbaric bupivacaine in the term parturient. Anesthesiology. 1990;72(3):478-82.

2. Norris MC. Height, weight, and the spread of subarachnoid hyperbaric bupivacaine in the term parturient. Anesth Analg. 1988;67(6):555-58.

3. Russell IF, Holmqvist EL. Subarachnoid analgesia for caesarean section. A double-blind comparison of plain and hyperbaric 0.5\% bupivacaine. Br J Anaesth. 1987;59(3):347-53.

4. Vercauteren MP, Coppejans HC, Hoffmann VL, Saldien V, Adriaensen HA. Small-dose hyperbaric versus plain bupivacaine during spinal anesthesia for cesarean section. Anesth Analg. 1998;86(5):989-93.

5. Hocking G, Wildsmith JA. Intrathecal drug spread. Br J Anaesth. 2004;93(4):568-78.

6. Ekeløf NP, Jensen $E$, Poulsen J, Reinstrup P. Weight gain during pregnancy does not influence the spread of spinal analgesia in the term parturient. Acta Anaesthesiol Scand. 1997;41(7):884-87. 
7. Harten JM, Boyne I, Hannah P, Varveris D, Brown A. Effects of a height and weight adjusted dose of local anaesthetic for spinal anaesthesia for elective Caesarean section. Anaesthesia. 2005;60(4):348-53.

8. Wei CN, Zhang YF, Xia F, Wang LZ, Zhou QH. Abdominal girth, vertebral column length and spread of intrathecal hyperbaric bupivacaine in the term parturient. Int J Obstet Anesth. 2017;31:63-7.

9. Wei $\mathrm{CN}$, Zhou $\mathrm{QH}$, Wang LZ. Abdominal girth and vertebral column length aid in predicting intrathecal hyperbaric bupivacaine dose for elective cesarean section. Medicine (Baltimore). 2017;96(34):e7905.

10. Gambus PL, Jaramillo S. Machine learning in anaesthesia: reactive, proactive... predictive!. Br J Anaesth. 2019;123(4):401-3.

11. Wijnberge M, Geerts BF, Hol L, et al. Effect of a Machine Learning-Derived Early Warning System for Intraoperative Hypotension vs Standard Care on Depth and Duration of Intraoperative Hypotension During Elective Noncardiac Surgery: The HYPE Randomized Clinical Trial. JAMA. 2020;323(11):1052-60.

12. Kendale $S$, Kulkarni $P$, Rosenberg $A D$, Wang J. Supervised Machine-learning Predictive Analytics for Prediction of Postinduction Hypotension. Anesthesiology. 2018;129(4):675-88.

13. Hashimoto DA, Witkowski E, Gao L, Meireles O, Rosman G. Artificial Intelligence in Anesthesiology: Current Techniques, Clinical Applications, and Limitations. Anesthesiology. 2020;132(2):379-94.

14. Gabriel RA, Sharma BS, Doan CN, Jiang X, Schmidt UH, Vaida F. A Predictive Model for Determining Patients Not Requiring Prolonged Hospital Length of Stay After Elective Primary Total Hip Arthroplasty. Anesth Analg. 2019;129(1):43-50.

15. Ousley R, Egan C, Dowling K, Cyna AM. Assessment of block height for satisfactory spinal anaesthesia for caesarean section. Anaesthesia. 2012;67(12):1356-63.

16. Hirabayashi Y, Shimizu R, Fukuda H, Saitoh K, Igarashi T. Soft tissue anatomy within the vertebral canal in pregnant women. Br J Anaesth. 1996;77(2):153-6.

17. Igarashi T, Hirabayashi $Y$, Shimizu R, Saitoh K, Fukuda H, Suzuki H. The fiberscopic findings of the epidural space in pregnant women. Anesthesiology. 2000;92(6):1631-6.

18. Takiguchi T, Yamaguchi S, Tezuka M, Furukawa N, Kitajima T. Compression of the subarachnoid space by the engorged epidural venous plexus in pregnant women. Anesthesiology. 2006;105(4):848-51.

19. Carpenter RL, Hogan QH, Liu SS, Crane B, Moore J. Lumbosacral cerebrospinal fluid volume is the primary determinant of sensory block extent and duration during spinal anesthesia. Anesthesiology. 1998;89(1):24-9.

20. Zhou QH, Shen $\mathrm{C}$, Chen $\mathrm{G}$. Abdominal girth and dorso-sacral distance can be used to estimate lumbosacral cerebral fluid volume. Acta Anaesthesiol Scand. 2018;62(2):234-41.

21. Ngan Kee WD, Khaw KS, Ng FF. Prevention of hypotension during spinal anesthesia for cesarean delivery: an effective technique using combination phenylephrine infusion and crystalloid cohydration. Anesthesiology. 2005;103(4):744-50.

22. Rout CC, Rocke DA, Levin J, Gouws E, Reddy D. A reevaluation of the role of crystalloid preload in the prevention of hypotension associated with spinal anesthesia for elective 
cesarean section [published correction appears in Anesthesiology 1994 Aug;81(2):529]. Anesthesiology. 1993;79(2):262-9.

Table 1 Clinical characteristics of term parturients

\begin{tabular}{lcccc}
\hline & Overall & $\begin{array}{c}\text { Appropriate } \\
\text { block }\end{array}$ & $\begin{array}{c}\text { Inappropriate } \\
\text { block }\end{array}$ & $P$ value \\
\hline No. pts (\%) & 684 & $516(75.44 \%)$ & $168(24.56 \%)$ & ---- \\
Age, yrs & $30.7 \pm 4.5$ & $30.7 \pm 4.5$ & $30.8 \pm 4.6$ & 0.717 \\
Weight, kg & $69.5 \pm 9.6$ & $69.1 \pm 9.4$ & $71.0 \pm 10.1$ & 0.022 \\
Height, cm & $159.3 \pm$ & $159.4 \pm 4.6$ & $159.1 \pm 4.8$ & 0.405 \\
$\begin{array}{l}\text { Vertebral column length, } \\
\text { cm }\end{array}$ & 4.7 & & & \\
Abdominal girth, cm & $99.9 \pm 7.1$ & $99.5 \pm 6.9$ & $101.2 \pm 7.7$ & 0.007 \\
Fundal height, cm & $35.2 \pm 2.8$ & $35.2 \pm 2.9$ & $35.2 \pm 2.6$ & 0.789 \\
$\begin{array}{l}\text { Fetal biparietal diameter, } \\
\text { cm }\end{array}$ & $9.1 \pm 0.5$ & $9.1 \pm 0.5$ & $9.2 \pm 0.4$ & 0.076 \\
Fetal weight, kg & $3.3 \pm 0.7$ & $3.4 \pm 0.7$ & $3.4 \pm 0.6$ & 0.445 \\
Bupivacaine dosage, $\mathrm{mL}$ & $2.0 \pm 0.2$ & $1.9 \pm 0.2$ & $2.0 \pm 0.1$ & 0.057 \\
\hline
\end{tabular}

Table 2 Parameter selection of lasso regression and model evaluation for different parameters

\begin{tabular}{|c|c|c|c|c|c|}
\hline$\lambda$ Value & $\begin{array}{l}\text { Selected physical } \\
\text { variable }\end{array}$ & $\begin{array}{l}\text { Weight for } \\
\text { variable }\end{array}$ & $\begin{array}{l}\text { Equation } \\
\text { Intercept }\end{array}$ & MSE & $R^{2}$ \\
\hline \multirow{2}{*}{0.055} & Abdominal girth & -0.017599 & \multirow{2}{*}{0.5922} & \multirow{2}{*}{0.0087} & \multirow{2}{*}{0.8070} \\
\hline & Vertebral column length & 0.055117 & & & \\
\hline \multirow{3}{*}{0.038} & Height & 0.000141 & \multirow{3}{*}{0.5071} & \multirow{3}{*}{0.0086} & \multirow{3}{*}{0.8108} \\
\hline & Abdominal girth & -0.018174 & & & \\
\hline & Vertebral column length & 0.057225 & & & \\
\hline \multirow{4}{*}{0.031} & Height & 0.000286 & \multirow{4}{*}{0.4516} & \multirow{4}{*}{0.0085} & \multirow{4}{*}{$\begin{array}{c}0.8119 \\
9\end{array}$} \\
\hline & Weight & -0.000171 & & & \\
\hline & Abdominal girth & -0.018232 & & & \\
\hline & Vertebral column length & 0.058107 & & & \\
\hline
\end{tabular}




\begin{tabular}{|l|l|c|c|c|c|}
\hline \multirow{3}{*}{0.020} & Height & 0.000618 & & \\
\cline { 2 - 3 } & Weight & -0.000600 & & \\
\cline { 2 - 3 } & Fundal height & -0.001253 & & 0.3687 & 0.0084 \\
4 & 0.8132 \\
& Abdominal girth & -0.017910 & & \\
\cline { 2 - 3 } & Vertebral column length & 0.059371 & & & \\
\hline
\end{tabular}

MSE: Mean-square error; $R^{2}$ : Coefficient of determination

\section{Figure legends}

Fig. 1 Actual and predicted bupivacaine volume for validation cohorts with 104 term parturients for validation; the ordinate is bupivacaine volume and abscissa is abdominal girth.

Fig. 2 Actual and predicted bupivacaine volume for validation cohorts with 104 term parturients for validation; the ordinate is bupivacaine volume and abscissa is vertebral column length. 
Figures

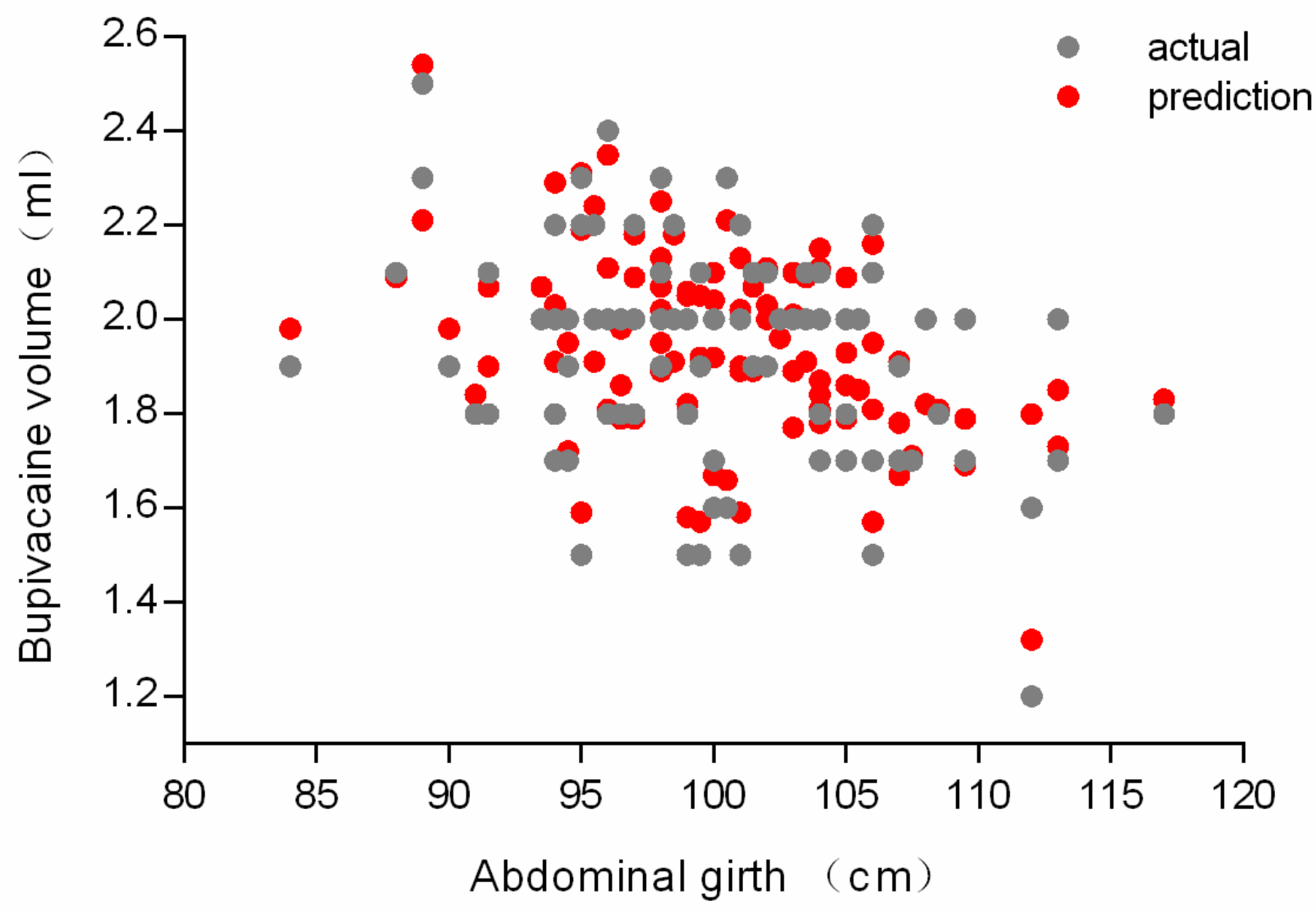

Figure 1

Actual and predicted bupivacaine volume for validation cohorts with 104 term parturients for validation; the ordinate is bupivacaine volume and abscissa is abdominal girth. 


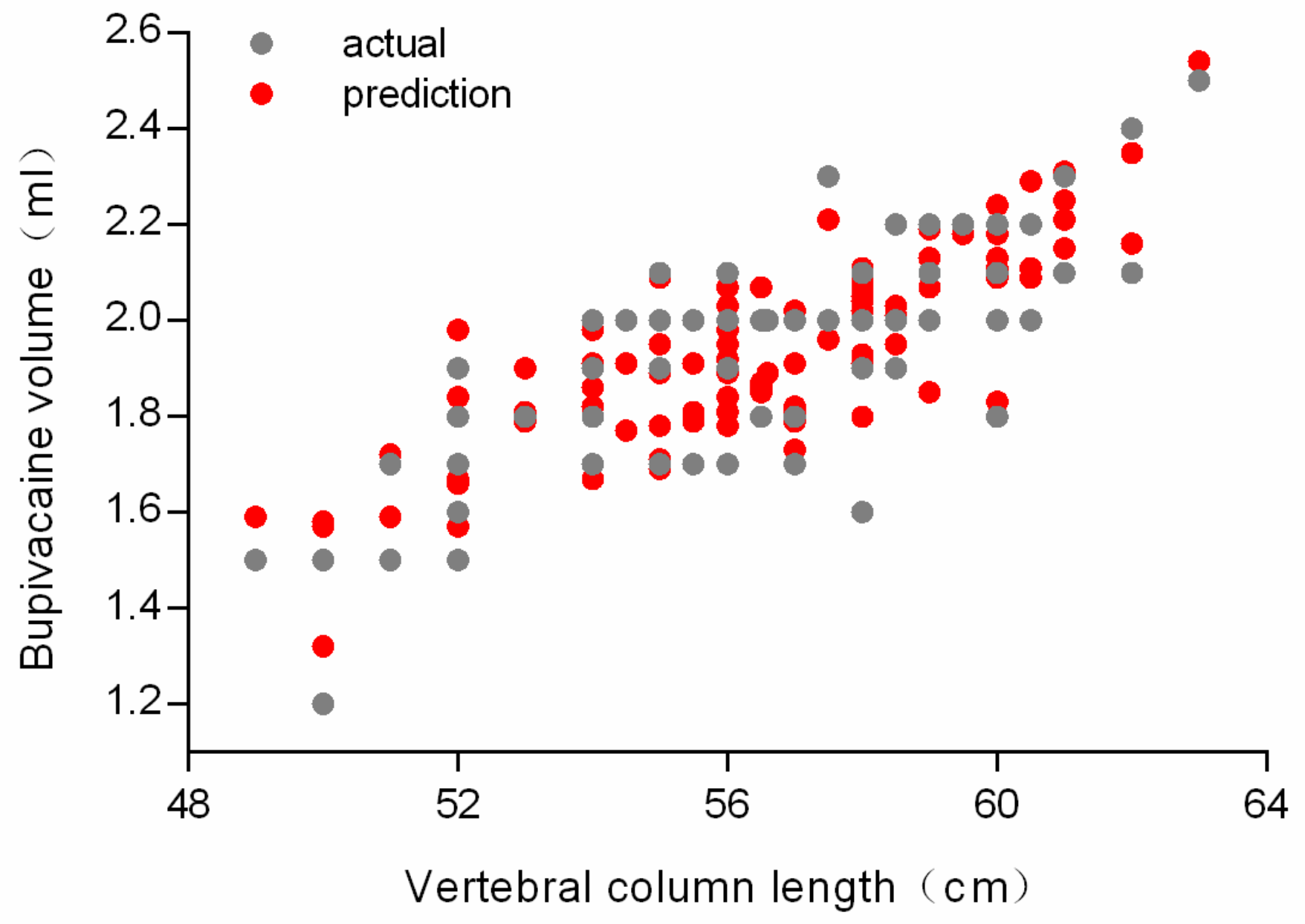

Figure 2

Actual and predicted bupivacaine volume for validation cohorts with 104 term parturients for validation; the ordinate is bupivacaine volume and abscissa is vertebral column length.

\section{Supplementary Files}

This is a list of supplementary files associated with this preprint. Click to download.

- Supplementaryfile.pdf 